\title{
Whole-Heart Cine MRI in a Single Breath-Hold - A Compressed Sensing Accelerated 3D Acquisition Technique for Assessment of Cardiac Function
}

\section{Cine-MRT mit vollständiger Herzabdeckung in einem Atemstopp - Eine Compressed-Sensing beschleunigte 3-D-Akquisitionstechnik zur Bestimmung der Herzfunktion}

Authors

Affiliations
T. Wech ${ }^{1,2}$, W. Pickl' , J. Tran-Gia' ${ }^{1}$, C. Ritter ${ }^{1}$, M. Beer ${ }^{1,3}$, D. Hahn' ${ }^{1}$, H. Köstler ${ }^{1,2}$

Institute of Radiology, University of Würzburg

Comprehensive Heart Failure Center, University of Würzburg

3 University Graz, University Hospital of Radiology
Key words

O heart

- MR imaging

- MR functional imaging

received $\quad 17.4 .2013$

accepted 19.7.2013

Bibliography

Dol http://dx.doi.org/

10.1055/s-0033-1350521

Published online: 30.8.2013

Fortschr Röntgenstr 2014; 186 :

37-41 @ Georg Thieme Verlag

KG Stuttgart · New York ·

ISSN 1438-9029

\section{Correspondence}

\section{Tobias Wech}

Institut für Röntgendiagnostik, Universität Würzburg Oberdürrbacher Straße 6 97080 Würzburg

Germany

Tel.: ++49/931/20134750

Fax: ++49/931/20134209

wech@roentgen.uni-

wuerzburg.de

\section{Zusammenfassung}

$\nabla$

Ziel: Ziel dieser Studie war es, die funktionelle MR-Herzbildgebung mit vollständiger Herzabdeckung mittels der Kombination aus einer unterabgetasteten 3-D-Aufnahmetechnik und einer Compressed-Sensing-Rekonstruktion in einem Atemstopp durchzuführen.

Material und Methoden: Die Messungen wurden mittels einer SSFP-Sequenz an einem 3T-Ganzkörpersystem, das mit einer 32-Kanalspule ausgestattet ist, durchgeführt. Um eine möglichst effiziente Unterabtastung des k-Raumes und damit eine Beschleunigung der Datenakquisition zu ermöglichen, wurde eine radiale 3-D-Stack-ofStars-Trajektorie verwendet. Anschließend wurden die fehlenden Daten mittels Compressed-Sensing rekonstruiert. Zur Validierung der Technik wurden zunächst Simulationen anhand mehrerer standardmäßig an einem gesunden Probanden aufgenommener 2-D-Cine-Datensätze durchgeführt. Die erzielten Ergebnisse wurden auf systematische Fehler und Auflösungsverluste untersucht. Anschließend wurde mittels der Technik die funktionelle MR-Herzbildgebung des gesamten Herzens in einem Atemstopp von $27 \mathrm{~s}$ durchgeführt. Die entwickelte Untersuchungsmethode wurde an drei gesunden Probanden getestet, um die Reproduzierbarkeit zu kontrollieren.

Ergebnisse: Die Simulationen zeigten nur kleine systematische Fehler in der um einen Faktor von 10 beschleunigten und anschließend mit der vorgestellten Methode rekonstruierten Aufnahme (zeitliche Auflösung: $47 \mathrm{~ms}$, räumliche Auflösung: 1,4×1,4×8 mm, 3-D-Bildmatrix: $208 \times 208 \times 10$ ). In der Bildebene war ein leichter Auflösungsverlust von $15 \%$ zu verzeichnen. Auch die anschließende in-vivo-Studie (zeitliche Auflösung: $40.5 \mathrm{~ms}$, räumliche Auflösung: $2,1 \times 2,1 \times 8 \mathrm{~mm}$, 3-D-Bildmatrix: $224 \times 224 \times 12$ ) bestätigte die gute Bildqualität unter einer 10,7-fachen Beschleunigung.

\section{Abstract \\ $\nabla$}

Purpose: The aim of this study was to perform functional MR imaging of the whole heart in a single breath-hold using an undersampled $3 \mathrm{D}$ trajectory for data acquisition in combination with compressed sensing for image reconstruction.

Materials and Methods: Measurements were performed using an SSFP sequence on a $3 \mathrm{~T}$ wholebody system equipped with a 32-channel body array coil. A $3 \mathrm{D}$ radial stack-of-stars sampling scheme was utilized enabling efficient undersampling of the k-space and thereby accelerating data acquisition. Compressed sensing was applied for the reconstruction of the missing data. A validation study was performed based on a fully sampled dataset acquired by standard Cartesian cine imaging of $2 \mathrm{D}$ slices on a healthy volunteer. The results were investigated with regard to systematic errors and resolution losses possibly introduced by the developed reconstruction. Subsequently, the proposed technique was applied for in-vivo functional cardiac imaging of the whole heart in a single breath-hold of $27 \mathrm{~s}$. The developed technique was tested on three healthy volunteers to examine its reproducibility.

Results: By means of the results of the simulation (temporal resolution: $47 \mathrm{~ms}$, spatial resolution: $1.4 \times 1.4 \times 8 \mathrm{~mm}, 3 \mathrm{D}$ image matrix: $208 \times 208 \times 10$ ), an overall acceleration factor of 10 has been found where the compressed sensing reconstructed image series shows only very low systematic errors and a slight in-plane resolution loss of $15 \%$. The results of the in-vivo study (temporal resolution: $40.5 \mathrm{~ms}$, spatial resolution: $2.1 \times 2.1 \times 8 \mathrm{~mm}, 3 \mathrm{D}$ image matrix: $224 \times 224 \times 12$ ) performed with an acceleration factor of 10.7 confirm the overall good image quality of the presented technique for undersampled acquisitions.

Conclusion: The combination of $3 \mathrm{D}$ radial data acquisition and model-based compressed sensing 
Schlussfolgerung: Die Kombination einer radialen 3-D-Aufnahme mit einer modell-basierten Compressed-Sensing-Rekonstruktionstechnik erlaubt hohe Beschleunigungsfaktoren und ermöglicht damit eine funktionelle Aufnahme mit vollständiger Herzabdeckung in nur einem Atemstopp. Die Bildqualität in den Simulations- sowie in-vivo-Ergebnissen unterstreicht das Potential der Technik für eine zeiteffizientere Bestimmung der funktionellen Herzparameter. reconstruction allows high acceleration factors enabling cardiac functional imaging of the whole heart within only one breathhold. The image quality in the simulated dataset and the in-vivo measurement highlights the great potential of the presented technique for an efficient assessment of cardiac functional parameters.

Citation Format:

- Wech T, Pickl W, Tran-Gia J etal. Whole-Heart Cine MRI in a Single Breath-Hold - A Compressed Sensing Accelerated 3D Acquisition Technique for Assessment of Cardiac Function. Fortschr Röntgenstr 2014; 186: 37-41

\section{Introduction}

$\nabla$

Multi-slice, multiple breath-hold ECG-gated cine magnetic resonance imaging (MRI) is the method of choice for the assessment of global and regional myocardial function [1 - 3]. However, the performance of this procedure requires one breath-hold of the patient for each of the $10-15$ slices, which are necessary to cover the entire heart. This deteriorates patient comfort and leads to long scan times. To achieve identical heart coverage in only one breath-hold, however, an acceleration of the data acquisition by a factor higher than ten is necessary.

In the last decade, a variety of different techniques has been proposed enabling data acquisition below the Nyquist sampling limit and thus considerably speeding up MRI measurements in general. Parallel Imaging (PI, [4]) exploits the coil sensitivity information obtained in phased-array coil acquisitions to reconstruct images from incompletely sampled datasets. Dedicated techniques have further been proposed especially for the accelerated acquisition of dynamic processes like the beating heart, e.g. AUTO-SENSE [5]. However, PI acceleration implicates a spatially variant noise enhancement which results in inadequate image quality for the acceleration factors needed.

Recently, the theory of compressed sensing (CS) has been introduced into MRI, also with the goal of a substantial reduction of scan times [6]. CS exploits the sparsity of the image data in any known transform domain to reconstruct undersampled k-spaces. Recent publications report on the successful application of CS in both preclinical as well as clinical MRI $[7,8]$.

Non-Cartesian data sampling has proven to improve k-space acquisition with an increased efficiency for certain applications. Although radial trajectories require more projections to fulfill the Nyquist criterion compared to a corresponding Cartesian sampling scheme, the technique yields artifacts of much higher incoherence in the case of undersampling. Exciting an entire slab of the object to be imaged and subsequently separating it by phase encoding steps (3 D MRI, [9]) substantially increases the signalto-noise ratio (SNR) with respect to consecutive single-slice acquisitions and additionally helps in avoiding misregistration between adjacent slices. Fourier interpolation can be used to determine continuous volumes, e.g. for modeling purposes.

The aim of this study was to perform whole-heart functional MRI in one breath-hold by using an accelerated non-Cartesian $3 \mathrm{D}$ acquisition scheme and a CS algorithm for image reconstruction. In a simulation, the developed reconstruction method was applied to retrospectively undersampled datasets and the results were compared to the complete dataset. Additionally, it was examined whether the proposed technique introduces systematic errors into the image series. As model-based reconstruction techniques such as CS may result in images suffering from blurring [10], a resolution study was performed.

Finally, the validated framework was used to acquire $3 \mathrm{D}$ cine series of the whole heart in single breath-holds of three healthy volunteers.

\section{Methods \\ $\nabla$}

All human studies were approved by the ethics committee of our institution and written informed consent was obtained from all volunteers participating in this study.

Data was sampled in an ECG-gated, segmented fashion as performed for conventional cine imaging ( $\bullet$ Fig. 1). A three dimensional k-space pattern, which will be referred to as "stack-ofstars" [11] in the following, was applied. The excited slab (z-direction) was phase-encoded as in standard $3 \mathrm{D}$ Cartesian acquisitions. In the two remaining directions $\left(\mathrm{k}_{\mathrm{x}}\right.$ and $\left.\mathrm{k}_{\mathrm{y}}\right)$, the sampling featured non-Cartesian radial projections. These radial sampling schemes were additionally twisted with respect to each other in the slab direction $\mathrm{k}_{\mathrm{z}}$ and in the temporal domain of consecutive heart phases. This maximizes the overall k-space coverage and the incoherence of the artifacts in image space and the temporal frequency domain in case of undersampling.

Image reconstruction was performed offline using Matlab (MathWorks, Natick, MA, see block diagram in • Fig. 2). Initially, the non-Cartesian data were shifted to a Cartesian grid using self-calibrating GROG [12]. Next, a compressed sensing (CS) algorithm was utilized to reconstruct the data not covered by the sampling scheme [13]. CS makes it possible to determine fully sampled datasets out of an undersampled k-space acquisition $\mathbf{k}$ by using prior knowledge about the object to be imaged. The prior knowledge in this work was represented by the sparsity of temporal difference images of the dynamic series [7]. The desired image space I was determined iteratively by consecutively thresholding the data $\left(\mathbf{S T}_{\mathrm{T}}\right)$ in the sparse transform domain $\Psi$ and reinserting the measured data $\mathbf{k}$ after every iteration $\mathbf{n}$ for consistency:

$\mathbf{I}_{n+1}=\Psi^{-1} \mathbf{S T}_{\mathrm{T}}\left[\Psi\left(\mathbf{I}_{n}+\mathbf{R}^{\mathrm{T}}\left(\mathbf{k}-\mathbf{R} \mathrm{I}_{n}\right)\right)\right]$ with $\mathrm{ST}_{\mathrm{T}}[\mathbf{c}]=(|\mathrm{c}|-\mathrm{T})_{+} e^{\mathrm{i} \text { angle(c) }}$

$\mathbf{R}$ denotes the operator which is transforming the image space into the undersampled k-space. It also performs the combination of images acquired by phased-array coils (decombination for $\mathbf{R}^{\mathbf{T}}$ ). In our study, the images where combined by the technique proposed by Walsh et al. [14] prior to each thresholding step and decombined for reinserting the measured data, thereby maximizing the SNR for the thresholding step.

The thresholding parameter t was tuned empirically to a value within the range of the noise level in the sparse domain. The 


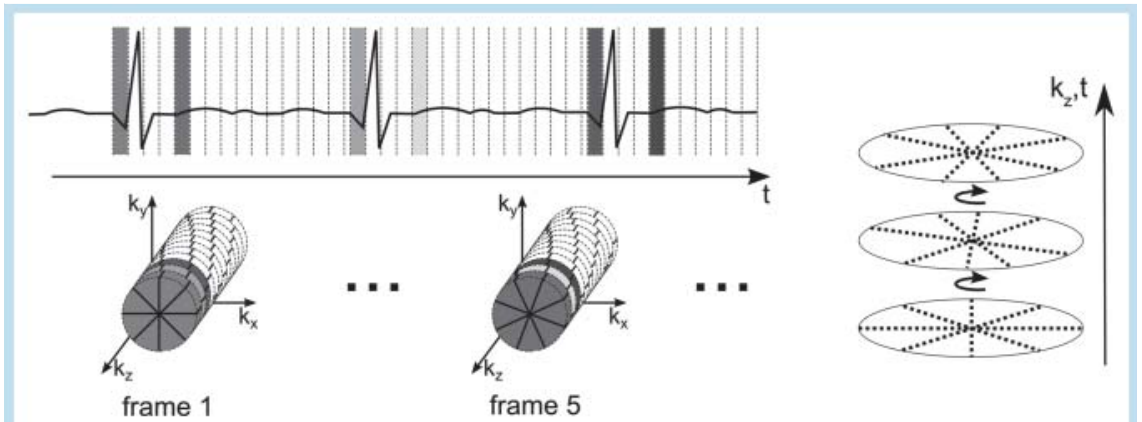

Fig. 1 Schematic illustration of the acquisition process: Data are sampled in a segmented fashion using an ECG-trigger. A $3 \mathrm{D}$ stack-of-stars is acquired for every time frame representing a single phase of the heart beat. The stars are twisted with respect to each other in the z-direction and the temporal domain in order to cover the k-space evenly.
Abb. 1 Schematische Illustration des Akquisitionsprozesses: Die Daten werden mittels eines EKG-Triggers segmentiert aufgenommen. Ein 3-D„Stack-of-Stars“ wird für jeden Zeitschritt akquiriert. Dieser repräsentiert eine Phase des Herzschlages. Das Sternmuster wird in z- und zeitlicher Richtung gegeneinander verdreht, um eine gleichmäßige Abdeckung zu erzielen.

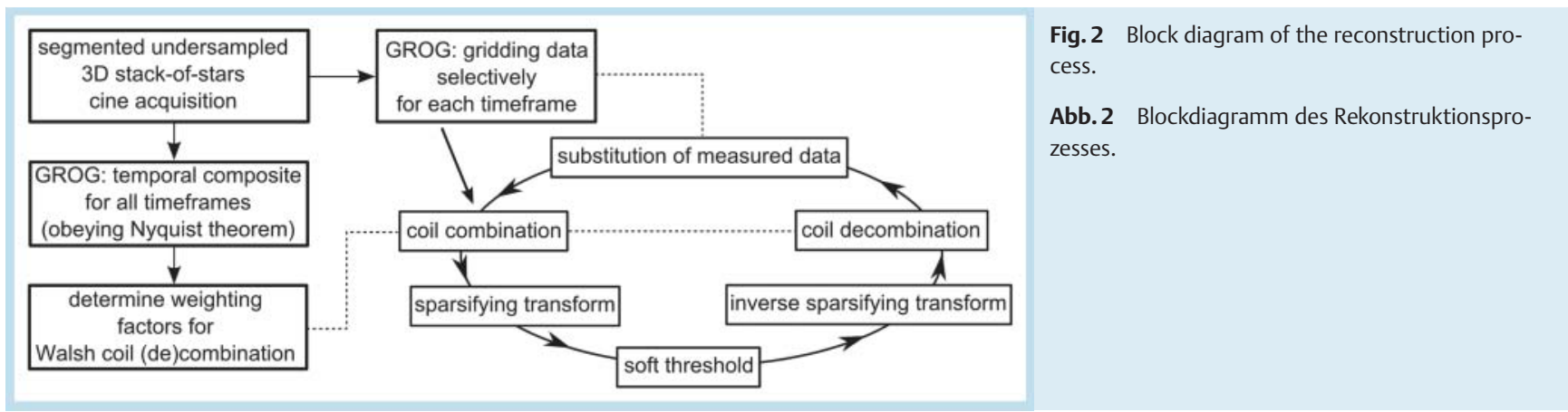

weighting factors for coil (de-)combinations were determined by gridding the data of all timeframes into one k-space.

\section{Simulation using a retrospectively undersampled dataset}

To evaluate the proposed concept, simulations were performed using data of a conventional Cartesian multi-slice $2 \mathrm{D}$ in-vivo cine examination using a short-axis view (Magnetom Trio, Siemens AG Healthcare Sector, SSFP, TR $3.8 \mathrm{~ms}$, FOV $380 \times 297 \mathrm{~mm}$, matrix size $256 \times 208,10$ consecutive slices, slice thickness $8 \mathrm{~mm}$, flip angle $45^{\circ}$, temporal resolution: $47 \mathrm{~ms}, 32$ channel body array coil, healthy volunteer). The fully sampled dataset was transformed into a $3 \mathrm{D}$ k-space for each timeframe and cropped to a matrix size of $208 \times 208 \times 10$ representing a squared FOV in the $x-y$ plane (size $297 \times 297 \mathrm{~mm}$, spatial resolution of 3D image space: $1.4 \times 1.4 \times 8 \mathrm{~mm}$ ). A pseudo radial stack-of-stars pattern (i. e., radial projections approximated on a Cartesian grid) was applied to retrospectively simulate a dataset undersampled by a factor of $\mathrm{R}=10.9$ with respect to a stack-of-stars pattern within the radial Nyquist limit: 30 radial projections were sampled in each of the 10 partitions. The proposed algorithm was then applied to reconstruct the missing data and the results were compared to the fully sampled reconstruction. As a failing CS reconstruction can lead to systematic errors, a subtraction image was determined between the CS-reconstructed cine series and a reference reconstructed from a Nyquist-sampled stack-of-stars. It has been shown earlier [10] that CS-reconstructed images can be subject to spatial or temporal blurring which is hardly recognizable in a subtraction image.
Therefore, local point spread functions [10] were determined at 70 positions randomly distributed across the heart. The width of their main-lobes was used to examine the resolution in all three spatial dimensions and the temporal domain.

\section{$3 \mathrm{D}$ cine measurement in a single breath-hold}

In addition, an ECG-triggered segmented 3D SSFP sequence using the proposed undersampling scheme was implemented and in-vivo measurements were performed in expiration. The acquisition was repeated for three healthy volunteers in total to check reproducibility. One representative acquisition is presented in this manuscript (Magnetom Trio, Siemens AG Healthcare Sector, SSFP, TR $3.8 \mathrm{~ms}$, FOV $480 \times 480 \times 96 \mathrm{~mm}, 12$ partitions, 33 radial projections per partition, 224 read-out points, flip angle $29^{\circ}$, temporal resolution: $40.5 \mathrm{~ms}$, spatial resolution: $2.1 \times 2.1 \times 8 \mathrm{~mm}, 32$ channel body array coil). The acceleration factor thereby corresponded to $\mathrm{R}=10.7$ with respect to a fully sampled radial stack-of-stars acquisition and to $R=6.8$ with respect to a Cartesian $3 \mathrm{D}$ acquisition on the according grid, resulting in a total acquisition time of $27 \mathrm{~s}$. A trajectory correction [15] was applied to the acquired data and the CS algorithm described above was used to reconstruct missing data points. 


\section{Results and Discussion}

$\nabla$

\section{Simulation using a retrospectively undersampled} dataset

- Fig. 3 shows the results of the simulations. The first series ( 0 Fig. 3a) shows the conventional reconstruction of the fully sampled cine images of a human heart in end-systole. While only every second image of the z-direction is shown, the image quality was equivalent throughout the entire stack as well as in the temporal domain. The series in 0 Fig. $\mathbf{3 b}$ represents the corresponding data decimated by the undersampled pseudo stackof-stars trajectory and reconstructed using the proposed CS-procedure. The algorithm was capable of removing streaking and spatial blurring artifacts induced by the highly undersampled acquisition pattern. The CS-reconstructed images are hardly differ-
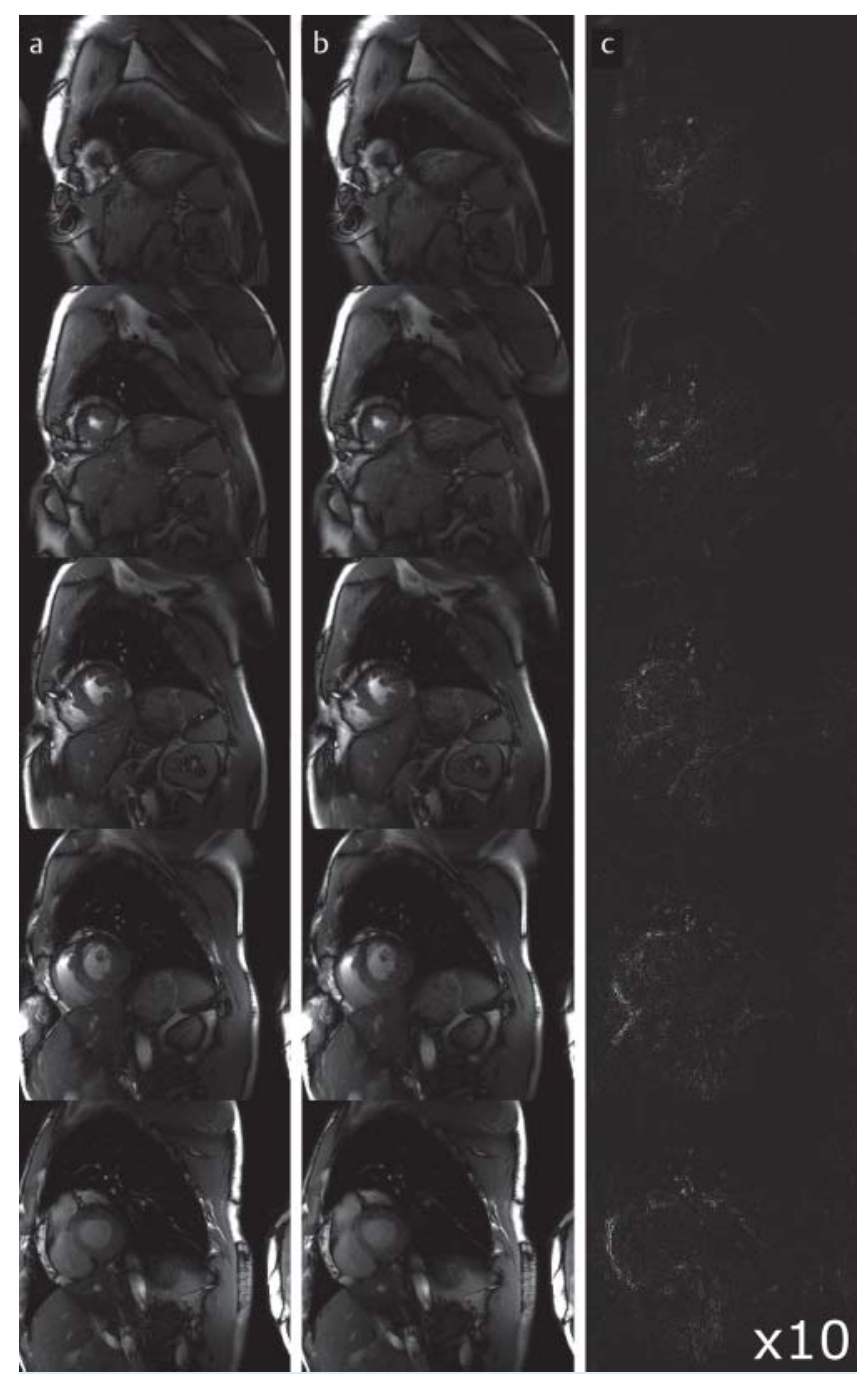

Fig. 3 Results of the simulated reconstruction. a Reference: Reconstruction of the fully sampled (stack-of-stars trajectory obeying the Nyquist criterion) data set. End-systolic frame. b CS-reconstruction of the retrospectively undersampled data $(R=10.9)$. End-systolic frame. c Subtraction image (b-a) multiplied by a factor of 10 .

Abb. 3 Ergebnisse der simulierten Rekonstruktion. a Vollständig abgetasteter (Stack-of-Stars-Trajektorie nach dem Nyquist-Kriterium) Referenzdatensatz, endsystolische Herzphase b CS-Rekonstruktion des retrospektiv unterabgetasteten Datensatzes $(R=10,9)$. Endsystolische Herzphase c Subtraktionsbild (b-a) mit einem Faktor von 10 multipliziert. entiable from the fully sampled images by a visual examination. The papillary muscles as well as endocardial and epicardial borders are excellently depicted throughout the whole image series in both diastole as well as systole. No deterioration in SNR is recognizable with regard to the Nyquist-sampled reference.

- Fig. 3c shows the corresponding subtraction images between - Fig. 3a, b. For reasons of clarity, they were multiplied by a factor of ten. In general, the systematic errors are at a very low level. The largest intensity is located at the border of the right ventricle and the septum.

The determination of local point spread functions resulted in a loss in spatial resolution of $15 \pm 8 \%$ with respect to a fully sampled stack-of-stars trajectory in the $\mathrm{x}$-y plane, while the z-direction and the temporal domain were not significantly affected by any losses.

\section{$3 \mathrm{D}$ cine measurement in a single breath-hold}

- Fig. 4 depicts the results of the in-vivo study for one representative volunteer. End-systolic and end-diastolic frames are depicted for every partition of the CS-reconstructed dataset. The streakings were removed by the CS-algorithm and the series provides sharp endocardial and epicardial borders as necessary for a determination of functional parameters.

The outer partitions show aliasing from the opposite side of the excited slab. Very low through plane aliasing is visible for central slices in the bright fat tissue. The myocardial border is clearly distinguishable throughout all slices and timeframes.

Despite high acceleration factors, the image quality was good in the simulations as well as in the in-vivo study. Temporal blurring was not evident in the performed study while the in-plane resolution was affected by a loss, however at an acceptably low level of $15 \%$. No severe systematic errors could be observed such that in summary, a determination of functional parameters should not be impaired by the proposed method. A comprehensive validation study is subject to future work.

As a high SNR is advantageous for a robust CS reconstruction, the combination and decombination of single-coil images led to a significant improvement of the results compared to a coil-bycoil CS reconstruction. The additional information gathered by a
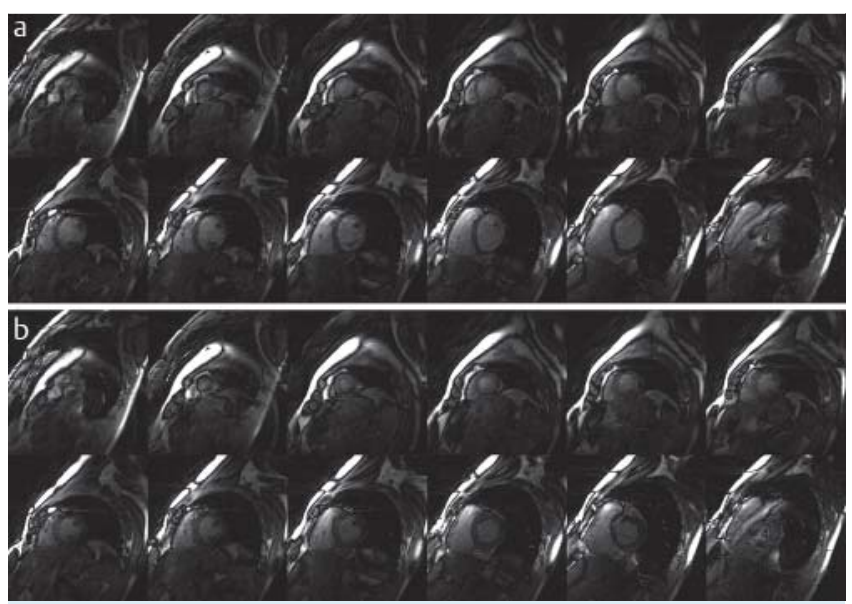

Fig. 4 Results of the in-vivo study of a 3D acquisition in a single breathhold. a End-diastolic frame. $\mathbf{b}$ End-systolic frame.

Abb. 4 Ergebnisse der in-vivo-Studie einer 3-D-Aufnahme in einem einzigen Atemstopp. a Enddiastolische Herzphase b Endsystolische Herzphase. 
multi-coil acquisition was therefore exploited, though not by PI acceleration. The overall post-processing in Matlab lasted 30 min. on a standard PC equipped with an Intel Core i7 CPU @ $3.4 \mathrm{GHz}$.

The stack-of-stars trajectory proved to be very suitable for a CS accelerated acquisition. The combination of phase encoding in the $\mathrm{z}$-direction and radial projections in each partition features undersampling artifacts of high incoherence and is thus ideal for CS. Twisting the radial patterns between partitions and timeframes allows even further enhancing of the incoherence compared to a mere repetition of the radial trajectory. A drawback in comparison to a Cartesian acquisition is the restricted choice of the field-of-view for the stack-of-stars technique. The radial patterns for the $x-y$-plane lead to quadratic slices, though a rectangular field of view would be more efficient for short-axis views. The aliasing of fat tissue mainly in outer partitions is a consequence of the imperfect slice profile of the fast excitation pulses used but does not impair an examination of the heart.

The spatial resolution $(2.1 \mathrm{~mm} \times 2.1 \mathrm{~mm} \times 8 \mathrm{~mm})$ of the in-vivo images is slightly lower than the resolution usually achieved by the gold standard in the clinical routine. However, it has been shown earlier that this resolution is sufficient for the determination of functional parameters [16]. The in-vivo images were acquired with a comparably low flip angle of $29^{\circ}$ which is a tradeoff between contrast, slice profile and SAR-limits.

Recently, a similar method has been proposed by Nam et al. [17]. There, a combination of a $3 \mathrm{D}$ acquisition and a CS algorithm is likewise applied to perform whole-heart imaging. The authors report on implementation of the demanding image reconstruction on a graphics processing unit (GPU) to substantially drive down the post-processing time. In contrast to our procedure, data was acquired using a respiratory navigator resulting in acquisition times longer than five minutes. Moreover, the images of each coil were reconstructed separately leading to a lower SNR for the thresholding iterations and thereby to a lower potential for acceleration. Combining the single breath-hold technique used in our studies with a GPU implementation of the reconstruction would allow fast data acquisition and post-processing directly at the scanner.

Earlier implementations of whole-heart functional imaging in a single breath-hold made use of an acceleration by reconstructing undersampled data by means of a previously acquired training dataset [18]. While this leads to additional scan time and the restriction that the cine data needs to match the training data, the method presented in this work does not use any pre-scans.

The regularly sampled slab ( $\mathrm{z}$ ) direction is predestined for an additional acceleration by PI. Although images are acquired with a 32-channel phased-array coil, the additional information is only exploited in the coil combination/decombination steps of the IST-algorithm. The implementation of a technique using both PI and CS is a work in progress and may further increase spatial and temporal resolution or shorten the acquisition time in order to make the technique applicable to the clinical routine.

Furthermore, the aliasing in the outer slices caused by the nonrectangular slab-selective excitation pulse may be reduced by using non-Cartesian density-weighted sampling in the $\mathrm{k}_{\mathrm{z}}$-direction as proposed by Gutberlet et al. [9]. Even if the k-space is undersampled in the outer parts for these patterns, the resulting artifacts would be more incoherent and could thus improve the image quality.

\section{Conclusion}

$\nabla$

In this work, a new technique to image the myocardial function of the entire heart in a single breath-hold is presented. Employing a $3 \mathrm{D}$ stack-of-stars undersampling pattern and a compressed sensing image reconstruction, an acceleration factor of higher than 10 could be achieved without compromising the diagnostic relevance of cardiac imaging at $3 \mathrm{~T}$. This corresponds to an extreme speed-up of the investigation with respect to the current gold standard of acquiring only one slice per breath-hold and therefore an improvement of patient comfort and throughput.

\section{References}

1 Lotz J, Kivelitz D, Fischbach $R$ et al. Empfehlungen fur den Einsatz der Computertomografie und Magnetresonanztomografie in der Herzdiagnostik. Teil 2 - Magnetresonanztomografie. Fortschr Röntgenstr 2009; 181: $800-814$

2 Lotz J, Barkhausen J, Gutberlet M. Kardiovaskuläre Bildgebung - interdisziplinär radiologisch. Fortschr Röntgenstr 2012; 184: 1093-1098

3 Wuest W, Machann W, Breunig F et al. Right ventricular involvement in patients with Fabry's disease and the effect of enzyme replacement therapy. Fortschr Röntgenstr 2011; 183: 1037-1042

4 Griswold MA, Jakob PM, Heidemann RM et al. Generalized autocalibrating partially parallel acquisitions (GRAPPA). Magn Reson Med 2002; 47: $1202-1210$

5 Köstler H, Beer M, Ritter C et al. Auto-SENSE view-sharing cine cardiac imaging. MAGMA 2004; 17: 63-67

6 Lustig M, Donoho D, Pauly JM. Sparse MRI: The application of compressed sensing for rapid MR imaging. Magn Reson Med 2007; 58: 1182 1195

7 Wech T, Lemke A, Medway D et al. Accelerating cine-MR imaging in mouse hearts using compressed sensing. J Magn Reson Imaging 2011; 34: 1072 - 1079

8 Wech T, Gutberlet M, Greiser A et al. High-resolution functional cardiac MR imaging using density-weighted real-time acquisition and a combination of compressed sensing and parallel imaging for image reconstruction. Fortschr Röntgenstr 2010; 182: 676-681

9 Jung BA, Hennig J, Scheffler K. Single-breathhold 3D-trueFISP cine cardiac imaging. Magn Reson Med 2002; 48: 921 - 925

10 Wech T, Stab D, Budich JC et al. Resolution evaluation of MR images reconstructed by iterative thresholding algorithms for compressed sensing. Med Phys 2012; 39: 4328-4338

11 Adluru G, Chen L, Kim SE et al. Three-dimensional late gadolinium enhancement imaging of the left atrium with a hybrid radial acquisition and compressed sensing. J Magn Reson Imaging 2011; 34: 1465 - 1471

12 Seiberlich N, Breuer F, Blaimer M et al. Self-calibrating GRAPPA operator gridding for radial and spiral trajectories. Magn Reson Med 2008; 59: 930-935

13 Khare $K$, Hardy CJ, King KF et al. Accelerated MR imaging using compressive sensing with no free parameters. Magn Reson Med 2012; DOI: $10.1002 / \mathrm{mrm} .24143$

14 Walsh DO, Gmitro AF, Marcellin MW. Adaptive reconstruction of phased array MR imagery. Magn Reson Med 2000; 43: 682 - 690

15 Wech T, Tran-Gia J, Hahn D et al. Iterative Trajectory Correction for Radial Projection Imaging. In: Annual Meeting of the ISMRM 2013: 129

16 Beer M, Stamm H, Machann W et al. Free breathing cardiac real-time cine MR without ECG triggering. International journal of cardiology 2010; 145: 380 - 382

17 Nam S, Akcakaya M, Basha T et al. Compressed sensing reconstruction for whole-heart imaging with 3D radial trajectories: A graphics processing unit implementation. Magn Reson Med 2012; DOI: 10.1002/ mrm.24234

18 Kozerke S, Tsao J, Razavi R et al. Accelerating cardiac cine 3D imaging using k-t BLAST. Magn Reson Med 2004; 52: 19-26 